\title{
Quercus canariensis Willd : variations saisonnières et stationnelles des teneurs en éléments minéraux des feuilles en Kroumirie (Tunisie)
}

\author{
B Hasnaoui, G Bonin*, S Garzino \\ Laboratoire de biosystématique et écologie méditerranéenne, université de Provence (Ura 1152), \\ FST Saint-Jérôme, case 421 bis, 13397 Marseille cedex 20, France
}

(Reçu le 7 novembre 1994 ; accepté le 24 juillet 1995)

\begin{abstract}
Résumé - Le suivi des nutriments a été réalisé dans les feuilles de Quercus canariensis Willd au cours d'un cycle annuel dans sept stations choisies le long d'un gradient bioclimatique en Kroumirie (Tunisie septentrionale). Les informations floristiques des stations (relevés phytosociologiques) ont permis, à l'aide de l'analyse factorielle des correspondances, de distinguer deux ensembles d'altitude et de conditions écologiques différentes. Les auteurs comparent l'évolution annuelle des principaux nutriments sur les feuilles de Quercus canariensis Willd dans le cadre de ces différentes entités écologiques. Les résultats soulignent l'importance des facteurs du milieu sur ces bilans. Ces résultats sont rapprochés de ceux connus sur d'autres chênes méditerranéens ou médioeuropéens. Si l'évolution des principaux nutriments suit la tendance générale constatée chez d'autres ligneux forestiers caducifoliés, le magnésium montre une trajectoire originale.
\end{abstract}

nutriments / Quercus canariensis Willd / variation spatiotemporelle / analyse multifactorielle / Tunisie

Summary - Seasonal nutrient dynamics in the Quercus canariensis Willd leaves. Impact of ecological situations in Kroumirie (Tunisia). Nutrient dynamics have been studied during 1 year in seven sites along a bioclimatic gradient in Kroumirie (Tunisia). Factorial correspondence analysis of floristic information has evidenced two pools characterized by different altitude and ecological factors. Seasonal evolutions of principal nutrient concentrations were compared, in Quercus canariensis leaves, in relation to these two different ecological units. This study stresses the strong relationship of environmental factors with this nutrient balance. The data have been compared with those recorded in other Mediterranean and medioeuropean oaks. The main nutrients follow the same tendancy as the other deciduous leaf forest trees. Only magnesium exhibits an original pattern.

nutrients / Quercus canariensis Willd / spatiotemporal analysis / factorial correspondence analysis / Tunisia

\footnotetext{
* Correspondance et tirés à part.
} 


\section{INTRODUCTION}

Le bilan nutritionnel du végétal par analyse chimique des feuilles est depuis très longtemps utilisé en agronomie (Lagatu et Maune, 1929 ; Prevot et Ollagnier 1956 ; Smith, 1962 ; Levy, 1968 ; Gautier, 1968).

Les espèces sylvatiques ont fait l'objet de nombreuses études sur les variations saisonnières des nutriments (Lowry et Avard, 1968 ; Leroy, 1968 ; Garbaye, 1972 ; Le Tacon et Toutain, 1973 ; Touzet, 1973), mais toujours dans le cadre de forêts de régions médioeuropéennes entretenues pour la production de bois.

Dans les sylves méditerranéennes dont I'histoire fut toujours perturbée, la situation n'est pas comparable à cause de l'hétérogénéité des peuplements forestiers, de l'impact humain souvent considérable et de la nature des espèces en présence.

Dans ces écosystèmes naturels forestiers, la nutrition minérale a été prise en compte pour l'étude des cycles biogéochimiques, quantifiant des flux d'éléments du sol au végétal puis du végétal au sol à travers plusieurs compartiments (Cole et Rapp, 1980).

L'étude présentée ici a pour objet de suivre les variations saisonnières des nutriments dans les feuilles de Quercus canariensis Willd (chêne zeen), espèce majeure des forêts de Kroumirie et du Maghreb en général, mais aussi de tenter d'analyser l'effet des conditions stationnelles sur ces bilans nutritionnels. C'est pourquoi cette essence forestière a été choisie comme matériel d'observation, le long d'un gradient écologique majeur.

Les formations sylvatiques de cette région méditerranéenne sont essentiellement colonisées par trois essences forestières $Q$ suber, $Q$ canariensis et $Q$ afares à un degré moindre. $Q$ canariensis occupe une place importante des faibles altitudes aux points culminants des massifs de l'intérieur en Kroumirie, sur les sols acides. Les forêts à $Q$ canariensis se rattachent sur le plan phytosociologique à deux classes:

- Quercetea ilicis $\mathrm{Br}-\mathrm{BI} 1947$ regroupant les forêts des étages thermo et méso méditerranéens ;

- Q pubescentis Doing-Kraft 1955 réunissant les groupements forestiers des étages supra et montagnard méditerranéens.

Dans ce large éventail de répartition bioclimatique, l'optimum d'abondance de cette essence forestière se situe vers 600 $1000 \mathrm{~m}$ d'altitude, là où le contexte lui est le plus favorable en Tunisie.

L'anthropisation excessive de ces milieux forestiers a perturbé la zonation altitudinale provoquant un mélange des essences forestières $Q$ suber et $Q$ canariensis Willd. Dans ce contexte de perturbation présentant une large palette de situations écologiques, on se propose de rechercher quel est le profil nutritionnel annuel le plus significatif, c'est-à-dire le plus représentatif de conditions écophysiologiques propices au bon développement du chêne zeen.

\section{MATÉRIELS ET MÉTHODES}

Sept sites ont été choisis pour cette étude. Leurs caractéristiques stationnelles et floristiques sont résumées dans le tableau I, leurs caractères édaphiques dans le tableau II.

Situés le long d'un gradient altitudinal, donc bioclimatique, ils correspondent à des milieux forestiers variés, ouverts ou fermés, où $Q$ canariensis présente des abondances diverses.

À partir de l'information écologique apportée par la composition floristique des stations, deux ensembles de stations peuvent être distingués en concordance avec les conditions bioclimatiques :

- L'un, de basse altitude, thermophile, à Myrtus communis toujours présent, correspond à des milieux situés en zone littorale (St 5, 6 et 7 ).

- L'autre, d'altitude moyenne beaucoup plus élevée $(900 \mathrm{~m})$ avec un cortège floristique où apparaissent $Q$ afares, Crataegus azerolus et Pulicaria odora, espèces de milieux plus frais, en zone continentale (St1, 2, 3 et 4).

Le bilan nutritionnel du peuplement de chaque station a été réalisé par prélèvements de feuilles sur cinq arbres représentatifs de l'arbre moyen, 
Tableau I. Composition floristique des stations.

\begin{tabular}{lrrrrrrr}
\hline & St 7 & St 6 & St 5 & St 4 & St 3 & St 2 & St 1 \\
Surface $\left(\mathrm{m}^{2}\right)$ & 100 & 100 & 100 & 100 & 100 & 100 & 100 \\
Couverture arborescente $(\%)$ & 80 & 95 & 70 & 70 & 90 & 80 & 80 \\
Couverture arbustive (\%) & 70 & 5 & 50 & 60 & 5 & 30 & 50 \\
Couverture herbacée (\%) & 5 & 5 & 10 & 40 & 30 & 20 & 25 \\
\hline
\end{tabular}

\begin{tabular}{|c|c|c|c|c|c|c|c|}
\hline Quercus canariensis Willd & 23 & 55 & 21 & 22 & 54 & 33 & 11 \\
\hline Quercus suber $\mathrm{L}$ & 11 & 11 & 33 & 12 & & 12 & + \\
\hline Myrtus communis $\mathrm{M}$ & 21 & 11 & 11 & & & & \\
\hline Erica arborea $\mathrm{L}$ & 22 & 12 & 22 & 12 & + & 11 & 33 \\
\hline Gallium ellipticum Willd & & + & & 11 & + & + & 11 \\
\hline Urginea maritima $\mathrm{M}$ et $\mathrm{W}$ & + & + & + & + & & & \\
\hline Agrimonia eupatoria $\mathrm{L}$ & & + & & 12 & & & \\
\hline Cyclamen africanum $B$ et $R$ & + & 11 & + & & & & \\
\hline Phillyrea media L & 11 & & 11 & & & & \\
\hline Arbutus unedo $L$ & 12 & & & & & 11 & + \\
\hline Asphodelus microcarpus S et $\mathrm{V}$ & & & & 11 & & 11 & + \\
\hline Quercus afares $\mathrm{L}$ & & & & & & 22 & 11 \\
\hline Cynosurus elegans Desf & + & + & & & & & \\
\hline Cytisus triflorus l'Herit & + & & & + & & & \\
\hline Cerastium pendadrum $\mathrm{L}$ & + & & & + & & + & \\
\hline Smilax aspera $\mathrm{L}$ & + & & & & & & \\
\hline Rubia peregrina $\mathrm{L}$ & + & & + & & & & \\
\hline Pistacia lentiscus $L$ & + & + & & & & & \\
\hline Daphne gnidium $\mathrm{L}$ & & & & & & + & \\
\hline Loniceras implexa Ait & + & + & & & & & \\
\hline Brachypodium distachyum PB & & + & & & & & \\
\hline Viburnum tinus $\mathrm{L}$ & + & & & & & & \\
\hline Euphorbia akenocarpa Guss & + & & & & & & \\
\hline Calycotome villosa & & & + & & & & \\
\hline Eryngium tricuspidatum $\mathrm{L}$ & & + & + & & & & \\
\hline Carex glauca Scop & & + & + & & & & \\
\hline Veronica agrestis $\mathrm{L}$ & & & & + & & & + \\
\hline Crataegus azarolus $\mathrm{L}$ & & & & + & & + & + \\
\hline Rubus ulmifolius Schott & + & & & + & & & + \\
\hline Fedia caput-bovii $\mathrm{Q}$ et $\mathrm{S}$ & & & + & + & & & + \\
\hline Pulicaria odora (L) Rchb & & & + & 12 & + & + & \\
\hline
\end{tabular}

Relevés phytosociologiques effectués sur $100 \mathrm{~m}^{2}$ par station.

situés en différents endroits de chaque station. Ces feuilles sont prises aux quatre orientations principales et au tiers moyen de la canopée, afin de gommer les variations intra-arbres. Le mélange permet d'obtenir une population de feuilles représentative de la réponse moyenne des arbres de la station.
Ce type de prélèvement a été effectué tous les deux mois afin de suivre un cycle annuel (du printemps à la fin de l'hiver suivant).

Pour chaque échantillon, sont pris en compte le poids frais et le poids sec. Les feuilles sont ensuite broyées pour obtenir une poudre homogène sur laquelle sont effectuées les analyses 


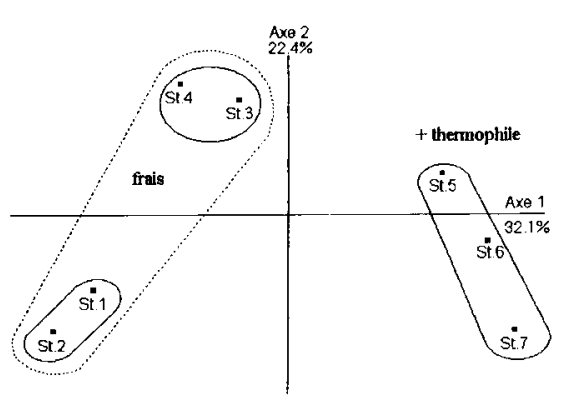

Fig 1. Plan $1 / 2$ de l'AFC sur les données floristiques.

chimiques. Le taux de nutriments retenu est exprimé en milligrammes par gramme de matière sèche.

Les concentrations en calcium et en magnésium ont été déterminées par spectrométrie d'absorption atomique et le potassium par spectrométrie d'émission de flamme après attaque sulfonitrique à chaud de $1 \mathrm{~g}$ de matériel séché et homogénéisé (Charlot, 1978). L'azote a été dosé suivant la classique méthode Kjeldahl.

Les résultats sont analysés sous plusieurs angles :

- en comparant l'évolution des différents éléments dans les stations au cours d'un cycle annuel ;

- en ordonnant les stations et les variables entre elles par le biais d'analyses multivariées (ACP : analyse en composantes principales) afin de souligner quels sont les nutriments marquant les différences entre stations et à quelles saisons :

- en confrontant ces résultats à ceux de l'information sur les données floristiques.

\section{RÉSULTATS}

\section{Discrimination des lots de stations}

Une ordination des stations, à l'aide d'une analyse factorielle des correspondances, a été réalisée à partir du cortège floristique utilisant le tableau constitué par les espèces en ligne et les relevés phytosociologiques en ordonnée. Cette ordination permet d'opposer de part et d'autre de l'axe 1 les stations de basse altitude et celles des zones plus élevées, distantes de la mer.

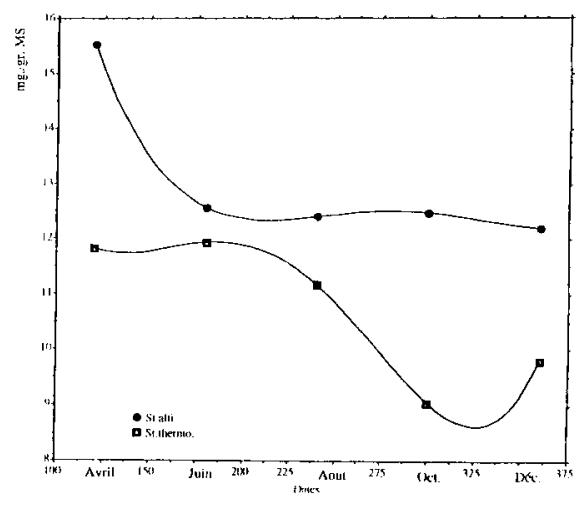

Fig 2. Courbe théorique (régression d'ordre 3) de la variation des teneurs en azote dans les deux ensembles de stations.

L'axe 2 discrimine les stations des deux sites continentaux alors que l'axe 3 dissocie les milieux forestiers ouverts des milieux fermés (fig 1).

Le plan 1-2 révèle un effet Guttman représentif d'un gradient de mésophilie croissante de la station 7 aux stations 1 et 2 .

Il convient d'ajouter, à la variable mésophilie relative des sylves, la perturbation anthropique plus ou moins grande de ces milieux.

L'examen des données sur les bilans de nutriments tiendra compte de la séparation objective en deux ensembles de stations (littorales et continentales) telle qu'elle apparaît à l'issue de ce premier traitement numérique.

\section{Variations saisonnières des principaux nutriments}

\section{L'azote}

Dans les stations d'altitude, le taux moyen de printemps est fort $(14,67 \mathrm{mg} / \mathrm{g}$ de matière sèche). Il baisse brutalement au début du cycle saisonnier pour se stabiliser ensuite jusqu'à l'hiver (tableau III et fig 2). La situation est très différente dans les stations de basse altitude avec une chute tardive de la teneur en azote des feuilles au cours de 
Tableau III. Variations du taux d'azote dans les stations d'altitude (en $\mathrm{mg} / \mathrm{g}$ de matière sèche).

\begin{tabular}{lcccccc}
\hline Dates & Station 1 & Station 2 & Station 3 & Station 4 & Moyenne & Ecart type \\
\hline Avril & 12,10 & 16,97 & 12,60 & 17,00 & 14,67 & 2,32 \\
Juin & 10,40 & 14,60 & 11,30 & 13,90 & 12,55 & 1,75 \\
Août & 11,50 & 12,20 & 13,40 & 12,50 & 12,40 & 0,68 \\
Octobre & 11,00 & 12,50 & 14,40 & 12,00 & 12,48 & 1,23 \\
Décembre & 11,40 & 10,80 & 13,10 & 13,50 & 12,20 & 1,13 \\
\hline
\end{tabular}

l'été et de l'automne, puis une légère remontée en hiver. Les réponses des feuillages des stations illustrent deux stratégies bien différentes dans l'utilisation de l'azote.

Le test $\mathrm{U}$ de Mann-Whitney $(p=0,014)$ démontre une différence significative tout au long de l'année entre ces deux réponses. La comparaison pour chaque période de prélèvement confirme ce résultat puisqu'en avril, août, octobre et décembre la différence est significative (tableau IV).

Le décalage est peut-être lié à l'ouverture du couvert forestier, les forêts ouvertes ayant leur optimum en juin-juillet (fig 2).

Dans les stations de moindre altitude (tableau V), les quantités d'azote dans les feuilles sont plus faibles et les variations entre stations moins grandes, avec un optimum de début d'été et une chute importante en début d'hiver.

La tendance générale d'une diminution du taux d'azote au cours de la vie de la feuille ne fait que confirmer le phénomène observé chez d'autres feuillus comme le hêtre ou le chêne pédonculé (Le Tacon et
Toutain 1973 ; Leroy, 1968). II faut cependant souligner que les taux d'azote chez le chêne zeen en Kroumirie sont nettement inférieurs à ceux d'autres feuillus répandus en Europe.

L'hétérogénéité des réponses du feuillage vis-à-vis de l'azote est plus grande en altitude, là où les conditions bioclimatiques sont pourtant plus favorables à l'espèce étudiée. Ceci peut sans doute être expliqué par la disparité des conditions stationnelles (pâturage en forêt et dégradation du couvert forestier).

Au cours du cycle annuel, c'est la fin du printemps qui révèle les plus grandes variations du taux d'azote, suivie de la période automnale. On peut donc estimer qu'il existe deux périodes d'activité physiologique, dont une très marquée en début d'été. La phase pré-hivernale est caractérisée par une grande homogénéité des valeurs en azote dans les feuilles.

Les courbes théoriques extrapolées des données moyennes des stations des deux ensembles illustrent, d'une part, la diffé-

Tableau IV. Test $U$ de Mann-Whitney soulignant les différences significatives entre les deux lots de stations.

\begin{tabular}{lccccc}
\hline Dates & Azote & Potassium & Calcium & Magnésium & $\%$ eau \\
\hline Avril & 0,0339 & NS & NS & NS & 0,0771 \\
Juin & NS & NS & NS & NS & 0,0339 \\
Août & 0,0771 & NS & NS & NS & 0,0771 \\
Octobre & 0,0339 & NS & 0,0339 & 0,0718 & 0,0339 \\
Décembre & NS & 0,0771 & 0,0771 & NS & NS
\end{tabular}


rence entre les deux situations et, d'autre part, la différence d'ampleur de la variation annuelle (fig 2).

\section{Le potassium}

Les teneurs en potassium dans les feuilles sont plus importantes dans les stations alticoles et atteignent leur optimum plus précocement au cours de la saison estivale (tableau $\mathrm{VI}$ ). Les différences de réponse des feuilles relevant des deux lots de stations à propos de cet ion ne sont pas significatives sauf en fin d'année (décembre, tableau IV).

Le potassium est fortement mobilisé dans les feuilles au moment de la phase de grande activité physiologique. Les réactions d'une station à l'autre sont très variables en altitude et moindres dans les zones plus thermophiles, avec des concentrations plus régulières sur le cycle annuel (tableau VII et fig 3 ).

On constate donc une grande similitude de situation entre les réponses de l'azote et du potassium dans les deux lots de stations (corrélation $r=0,74$ ). Ceci paraît normal puisque le rôle d'activateur général du

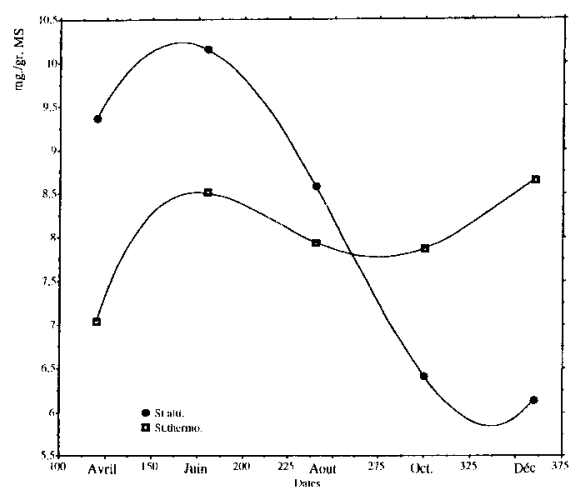

Fig 3. Courbe théorique (régression d'ordre 3) de la variation des teneurs en potassium.

métabolisme que joue $\mathrm{K}$ est bien établi, entraînant l'activation de l'assimilation d'azote.

Cependant, on peut s'étonner du retard constaté dans l'augmentation des taux d'azote et de potassium dans les feuilles des arbres de basse altitude, qui phénologiquement sont plus précoces.

La comparaison des valeurs obtenues sur $Q$ canariensis avec celles déjà publiées à propos de Fagus sylvatica (Le Tacon et

Tableau.V. Taux d'azote dans les stations thermophiles.

\begin{tabular}{lccccc}
\hline Dates & Station 5 & Station 6 & Station 7 & Moyenne & Écart type \\
\hline Avril & 11,86 & 12,02 & $11,, 60$ & 11,83 & 0,17 \\
Juin & 12,00 & 13,30 & 10,50 & 11,93 & $\mathbf{1 , 1 4}$ \\
Août & $\mathbf{1 1 , 2 0}$ & 11,60 & 10,70 & 11,17 & 0,39 \\
Octobre & 8,25 & 9,10 & 9,80 & 9,05 & 0,63 \\
Décembre & 9,20 & 11,40 & 8,80 & 9,80 & $\mathbf{1 , 1 4}$
\end{tabular}

Tableau VI. Taux de potassium dans les stations d'altitude.

\begin{tabular}{lrrcrrc}
\hline Dates & Station 1 & Station 2 & Station 3 & Station 4 & Moyenne & Écart type \\
\hline Avril & 6,05 & 10,71 & 7,97 & 10,90 & 9,36 & 1,96 \\
Juin & 10,06 & 14,03 & 7,39 & 8,89 & 10,09 & 2,50 \\
Août & 7,86 & 9,96 & 8,42 & 8,09 & 8,58 & 0,82 \\
Octobre & 7,46 & 5,10 & 6,45 & 6,64 & 6,41 & 0,85 \\
Décembre & 6,40 & 6,02 & 7,32 & 4,81 & 6,14 & 0,90 \\
\hline
\end{tabular}


Tableau VII. Taux de potassium dans les stations thermophiles.

\begin{tabular}{lccccc}
\hline Dates & Station 5 & Station 6 & Station 7 & Moyenne & Écart type \\
\hline Avril & 7,07 & 7,56 & 6,49 & 7,04 & 0,44 \\
Juin & 7,90 & 9,40 & 8,23 & 8,51 & 0,65 \\
Août & 7,97 & 6,08 & 9,77 & 7,94 & 1,51 \\
Octobre & 8,98 & 8,47 & 6,16 & 7,87 & 1,23 \\
Décembre & 9,39 & 10,01 & 6,55 & 8,65 & 1,50 \\
\hline
\end{tabular}

Tableau VIII. Taux de calcium dans les stations d'altitude.

\begin{tabular}{llccccc}
\hline Dates & Station 1 & Station 2 & Station 3 & Station 4 & Moyenne & Écart type \\
\cline { 2 - 5 } Avril & 5,80 & 6,40 & 6,60 & 5,20 & 6,00 & 0,55 \\
Juin & 5,78 & 4,19 & 6,57 & 5,62 & 5,54 & 0,86 \\
Août & 5,20 & 4,88 & 6,09 & 4,40 & 5,14 & 0,62 \\
Octobre & 6,26 & 9,12 & 9,55 & 6,05 & 7,74 & 1,59 \\
Décembre & 9,50 & 6,90 & 9,02 & 8,86 & 8,57 & 0,99 \\
\hline
\end{tabular}

Toutain) ou $Q$ pedunculata (Leroy) montre des taux en potassium sensiblement plus faibles sur le chêne zeen, mais l'écart est beaucoup moins important que dans le cas de l'azote.

\section{Le calcium}

Le calcium augmente de manière régulière au cours du vieillissement de la feuille. Dans les stations continentales, ce phénomène est plus sensible que dans les stations d'altitude inférieure. Dans le premier

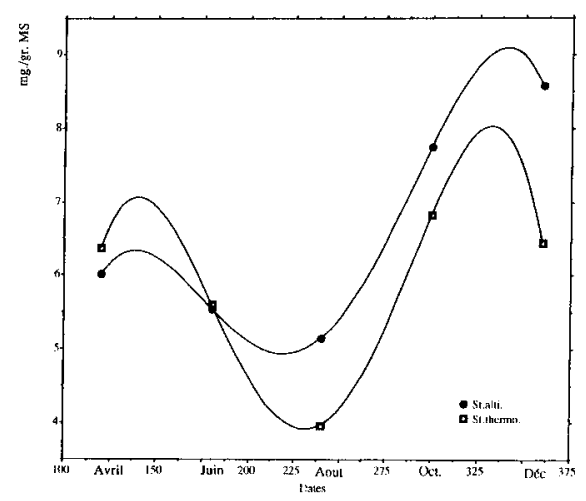

Fig 4. Courbe théorique (régression d'ordre 3) de la variation des teneurs en calcium. lot de stations, la quantité augmente d'un tiers dans un cycle annuel (tableau VIII), dans le second, elle ne fait que passer de 6,37 à $6,45 \%$ (tableau IX).

La différence de réponse entre les feuillages des deux ensembles de stations est significative en octobre et en décembre (tableau IV).

Le phénomène d'accumulation de cet ion en fonction du temps a déjà été observé chez les chênes méditerranéens à feuillage sclérophylle (Ed Derfoufi, 1986 ; Li-

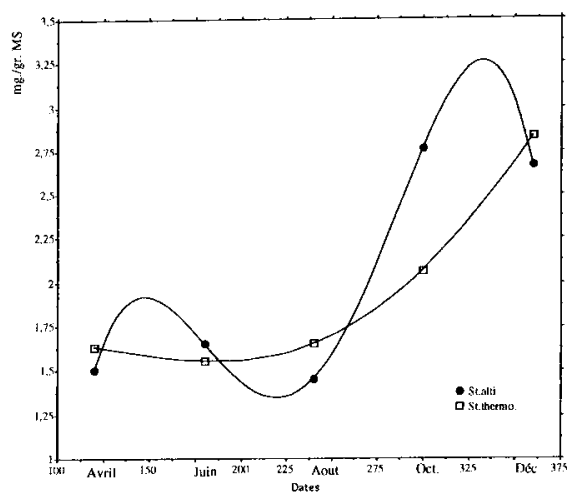

Fig 5. Courbe théorique (régression d'ordre 3) de la variation des teneurs en magnésium. 
Tableau IX. Taux de calcium dans les stations thermophiles.

\begin{tabular}{lccccc}
\hline Dates & Station 5 & Station 6 & Station 7 & Moyenne & Écart type \\
\hline Avril & 5,90 & 6,60 & 6,60 & 6,37 & 0,33 \\
Juin & 6,60 & 5,63 & 4,56 & 5,60 & 0,83 \\
Août & 4,00 & 3,60 & 4,24 & 3,95 & 0,26 \\
Octobre & 8,38 & 6,47 & 5,62 & 6,82 & 1,15 \\
Décembre & 7,95 & 5,30 & 6,10 & 6,45 & 1,11 \\
\hline
\end{tabular}

Tableau $\mathbf{X}$. Taux de magnésium dans les stations alticoles.

\begin{tabular}{lcccccc}
\hline Dates & Station 1 & Station 2 & Station 3 & Station 4 & Moyenne & Écart type \\
\hline Avril & 1,76 & 1,50 & 1,36 & 1,64 & 1,57 & 0,15 \\
Juin & 1,50 & 1,75 & 1,70 & 1,65 & 1,65 & 0,09 \\
Août & 1,55 & 1,65 & 1,40 & 1,20 & 1,45 & 0,17 \\
Octobre & 3,25 & 2,50 & 2,65 & 2,65 & 2,76 & 0,29 \\
Décembre & 2,50 & 2,50 & 2,85 & 2,80 & 2,66 & 0,16
\end{tabular}

vrelli, 1993), la figure 4 illustre ces variations. Ce phénomène avait été observé aussi chez le chêne pédonculé, avec des concentrations comparables de cet ion.

\section{Le magnésium}

Pour cet ion, le profil de variation des taux dans les quatre stations alticoles n'est pas comparable à celui des trois stations sous influence maritime.

Le premier groupe de stations montre un accroissement progressif du taux de magnésium, avec un premier maximum de début d'été suivi d'un creux de fin d'été et un second maximum automnal suivi du creux hivernal. L'influence du stress estival sur le bilan de cet ion est incontestable dans ces stations (tableau X).

Le second groupe de stations est caractérisé par un accroîssement progressif du taux de magnésium sans saisonnalité marquée. On assiste dans ce cas à un phénomène d'accumulation comme pour le calcium (tableau XI et fig 5).

Cette tendence générale est bien différente de celle observée par Leroy sur $Q$ pedunculata ou par Le Tacon sur $F$ sylvatica.
On peut s'interroger sur les raisons de ce mécanisme.

En conséquence, l'azote et le magnésium sont les deux paramètres dont les comportements au cours d'un cycle annuel différencient le plus les deux secteurs écologiques. Les taux de ces deux éléments sont donc particulièrement sensibles aux contraintes du milieu.

De manière générale, pour les stations continentales situées dans la zone considérée par les phytoécologues comme la zone potentielle du $Q$ canariensis, les bilans en nutriments sont plus élevés et les taux marquent des variations plus importantes au cours d'un cycle annuel. En zone sous influence maritime, les phénomènes sont atténués, comme si les échanges de nutriments se produisaient là au ralenti.

\section{Bilan de l'eau dans les feuilles}

Le contenu en eau des feuilles est maximal au printemps, marque un creux significatif durant la période estivale pour remonter au cours de l'automne. Ce profil annuel logique est identique dans les feuillages des stations d'altitude faible ou élevée. Cependant le phénomène de variation est ampli- 


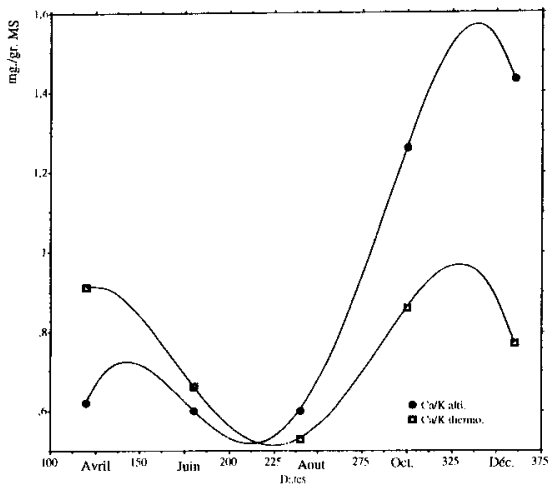

Fig 6. Courbe théorique (régression d'ordre 3) de la variation des teneurs saisonnières du rapport $\mathrm{Ca} / \mathrm{K}$.

fié dans les feuilles des chênaies des montagnes plus continentales, avec un maximum printanier de $60 \%$ d'eau et un creux estival très marqué. La saison sèche estivale est donc plus marquée sur le plan écophysiologique dans cette zone d'altitude (tableaux XII et XIII).

\section{Interrelations entre éléments minéraux}

L'examen comparé des courbes des figures 2 et 3 montre une assez bonne synergie entre potassium et azote, au niveau du feuillage des stations les plus thermophiles. Dans les stations continentales plus alticoles, ces relations sont statistiquement moins significatives et ne peuvent donc pas être prises en compte.

La relation entre potassium et calcium est illustrée par l'évolution du rapport $\mathrm{Ca} / \mathrm{K}$ (fig 6). Celui-ci tend à baisser dans la période estivale dans les deux ensembles de stations. La remontée automnale et hivernale est plus importante en zone thermophile, en

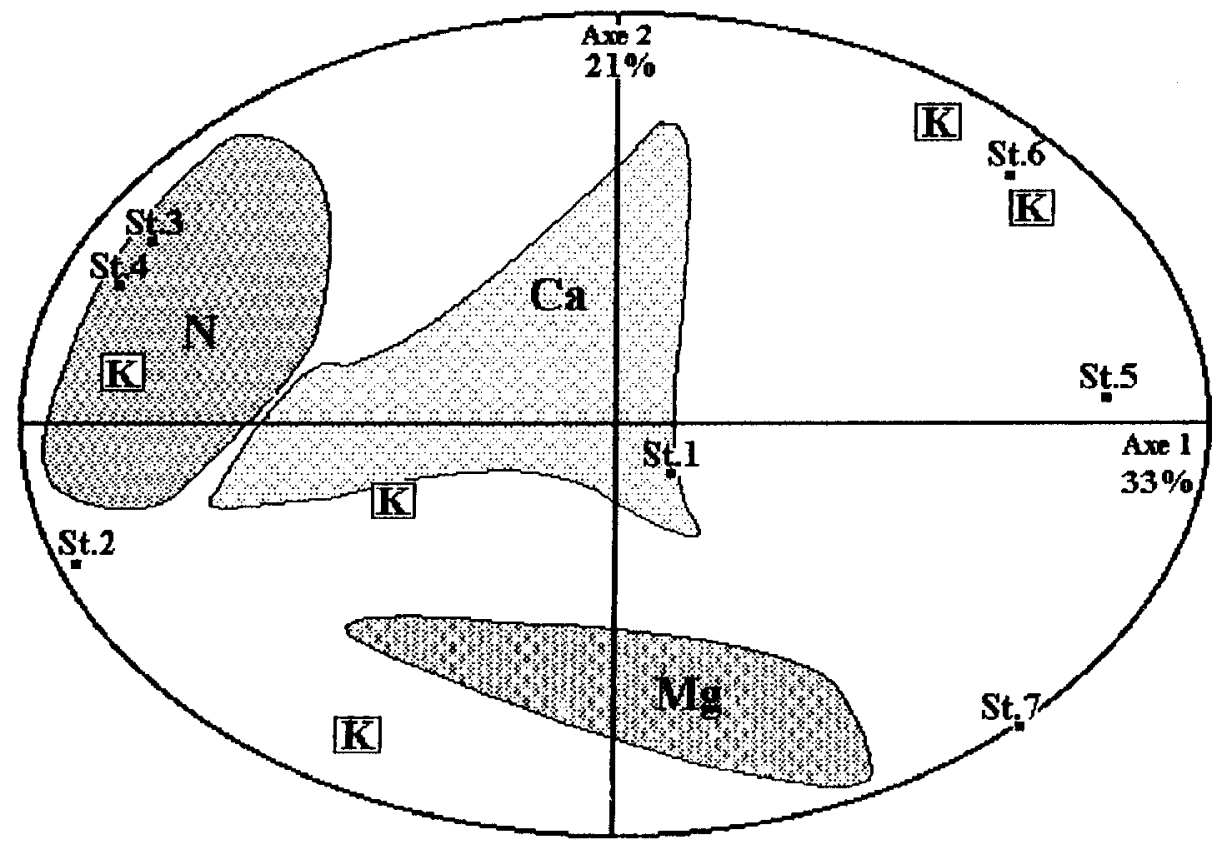

Fig 7. Plan $1 / 2$ de l'ACP de la totalité des données nutriments: nuages des points variables des éléments minéraux (schématisés) sauf pour $K$ et positions des stations. 
Tableau XI. Taux de magnésium dans les stations thermophiles.

\begin{tabular}{lccccc}
\hline Dates & Station 5 & Station 6 & Station 7 & Moyenne & Écart type \\
\hline Avril & 1,91 & 1,31 & 1,68 & 1,63 & 0,25 \\
Juin & 1,82 & 1,24 & 1,60 & 1,55 & 0,24 \\
Août & 1,65 & 1,40 & 1,90 & 1,65 & 0,20 \\
Octobre & 1,80 & 1,80 & 2,60 & 2,07 & 0,38 \\
Décembre & 2,80 & 2,30 & 3,40 & 2,83 & 0,45 \\
\hline
\end{tabular}

Tableau XII. Pourcentage d'eau dans les feuilles des stations alticoles.

\begin{tabular}{lcccccc}
\hline Dates & Station 1 & Station 2 & Station 3 & Station 4 & Moyenne & Écart type \\
\hline Avril & 5,97 & 36,15 & 57,93 & 67,44 & 62,06 & 4,19 \\
Juin & 37,84 & 13,30 & 34,81 & 31,89 & 34,46 & 2,55 \\
Août & 36,71 & 34,53 & 38,75 & 39,35 & 37,34 & 2,18 \\
Octobre & 49,66 & 46,16 & 47,13 & 44,42 & 46,84 & 2,19 \\
Décembre & 43,27 & 39,78 & 51,03 & 52,66 & 46,69 & 6,16 \\
\hline
\end{tabular}

raison à la fois de la plus forte importance du taux de calcium dans ce secteur durant cette période et du plus fort taux de potassium.

\section{Analyse globale des données sur un cycle annuel}

L'effet relatif et combiné des variables « nutriments » au cours du temps et dans les diverses stations peut être apprécié à l'aide de méthodes d'analyses multivariées comme l'analyse en composantes principales (ACP) qui permet de faire ressortir quelques tendances générales. Dans un premier temps, les données ont été traitées globalement, pour savoir si l'information apportée par les données nutriments aboutissait à une ordination comparable à celle obtenue à partir des données floristiques.

On peut constater une bonne concordance entre la position des stations sur la figure 1 (plan un demi de l'AFC des données floristiques) et celle des mêmes stations sur la figure 7 à l'issue du traitement des données nutriments, à l'exception de la station 1 qui occupe une position plus neutre, au centre du plan un demi de l'ACP.
Les conditions stationnelles, qui influencent de manière déterminante la composition floristique du groupement forestier, agissent de manière concordante sur la variation des nutriments et leur bilan peut donc constituer un bon élément de diagnostic écologique.

L'azote, toutes saisons confondues, marque le pôle négatif de l'axe 1 de l'ACP, caractérisant ainsi les stations alticoles. Le potassium de la période octobre-décembre est un élément caractéristique des stations thermophiles. À l'inverse, les points $\mathrm{K}$ correspondant à la période printanière marquent les stations alticoles. On voit donc qu'il y a dispersion des points représentant le potassium.

Le calcium ne semble pas déterminant dans cette analyse, cela ne signifie pas qu'il joue un rôle mineur mais que son importance dans la discrimination entre les deux grands ensembles écologiques forestiers est moindre.

\section{Traitement des données dans chaque ensemble de stations}

Le traitement des données de chaque ensemble écologique a été fait à partir d'une 
Tableau XIII. Pourcentage d'eau dans les feuilles des stations thermophiles.

\begin{tabular}{lccccc}
\hline Dates & Station 5 & Station 6 & Station 7 & Moyenne & Écart type \\
\hline Avril & 59,48 & 55,87 & 54,33 & 56,56 & 2,64 \\
Juin & 48,68 & 46,43 & 43,80 & 46,30 & 2,44 \\
Août & 38,76 & 43,19 & 39,60 & 40,52 & 2,36 \\
Octobre & 44,05 & 43,91 & 40,13 & 42,70 & 2,22 \\
Décembre & 52,53 & 55,31 & 44,07 & 50,64 & 5,85 \\
\hline
\end{tabular}

matrice dont les lignes (individus) sont les dates de prélèvement, et les colonnes sont les variables, taux des nutriments dans chaque station. Ceci permet de mettre en évidence les articulations entre nutriments dans les stations d'un même contexte écologique au cours du cycle annuel.

\section{Stations sous influence littorale thermophile}

La figure 8 est une représentation synthétique des dates de prélèvement (individus) et des nutriments (variables), qui met en évidence :

- la dispersion des points relatifs au potassium;

- le regroupement de points concernant l'azote vers le pôle négatif de l'axe 1 ;

- le regroupement de points concernant le magnésium au pôle positif du même axe ;

- la position intermédiaire et regroupée des points relatifs au calcium sur le cercle de corrélation, entre les pôles positifs des axes 1 et 2.

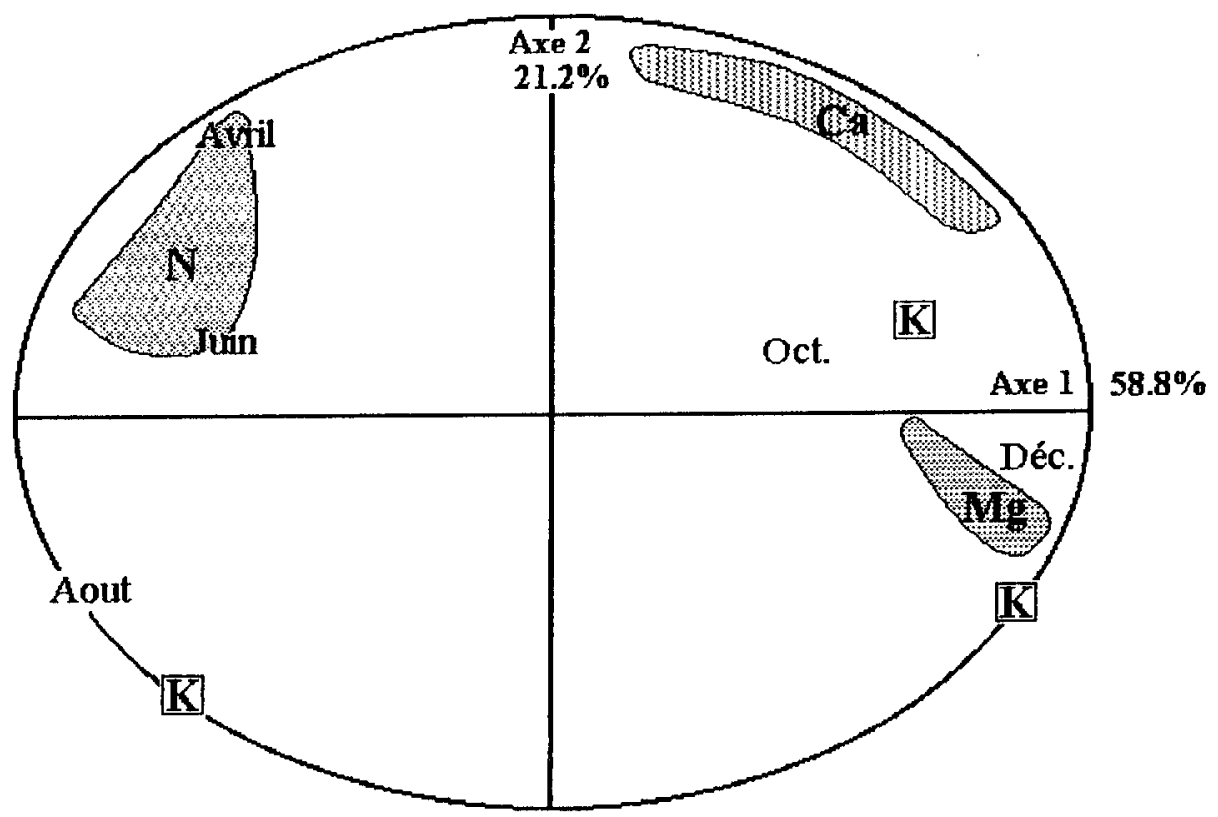

Fig 8. Plan $1 / 2$ de l'ACP relative aux données des stations thermophiles; cercle de corrélation des variables et positionnement des dates de prélèvement. 
On peut en tirer les conclusions suivantes:

- l'azote et le magnésium jouent un rôle déterminant de discrimination entre les périodes printemps-été et automne-hiver, bien marquées de part et d'autre de l'axe 1 qui représente $58 \%$ de l'information ;

- le calcium a un comportement assez homogène dans les trois stations thermophiles ;

- en revanche, le potassium présente une hétérogénéité certaine de réponse au cours du cycle saisonnier pour les trois stations thermophiles.

\section{Stations sous influence continentale}

La comparaison avec la situation précédente montre de grandes différences (fig 9 ). Les éléments déterminant l'axe 1 $(61,4 \%$ de l'information) sont le potassium (pôle négatif) et le magnésium (pôle positif), qui marquent l'opposition saisonnière entre avril-juin-août et octobre-décembre. L'azote ne joue pas un rôle majeur dans ce schéma, et le calcium est secondaire.

Ces deux analyses démontrent que la relation entre nutriments dans les feuillages des deux ensembles forestiers n'obéit pas aux mêmes équilibres. L'évolution saisonnière de chaque élément minéral étant différente d'un ensemble écologique à l'autre, il est normal que la combinaison des divers éléments apporte une réponse propre à chaque contexte écologique.

II convient cependant de souligner le fait que l'opposition entre la période printemps-été et la période automne-hiver se retrouve dans les deux ensembles écologiques, elle pourrait donc être prise comme une constante du chêne zeen.

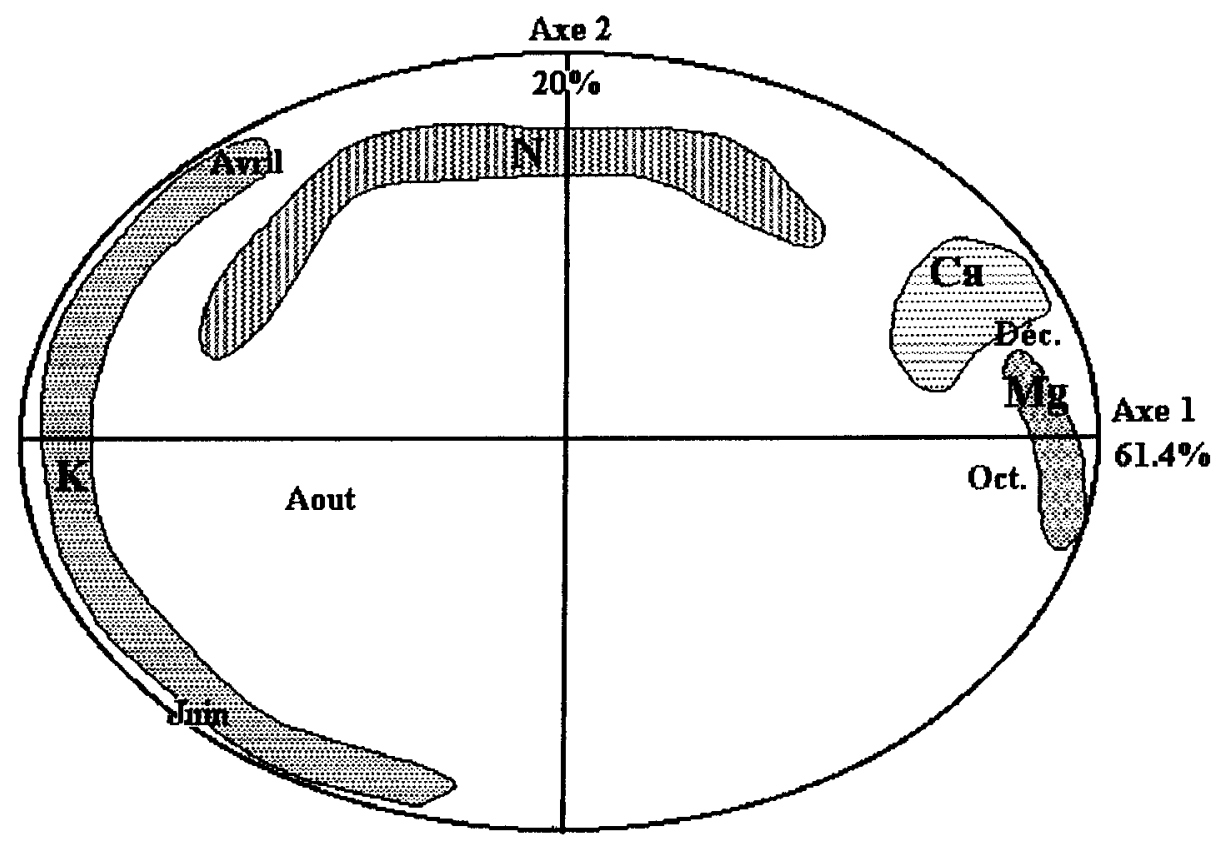

Fig 9. Plan 1/2 de l'ACP relative aux données des stations alticoles; cercle de corrélation des variables et positionnement des dates de prélèvement. 


\section{CONCLUSION}

$Q$ canariensis est considéré par les phytoécologues et biogéographes comme l'espèce dominante de la zone $500-1000 \mathrm{~m}$ dans les montagnes d'Afrique du Nord, zone qui correspond aux stations continentales de l'échantillon étudié.

Sa capacité de développement, de régénération, sa biomasse et l'occupation de l'espace en témoignent.

Les résultats présentés pourraient confirmer cette opinion dans la mesure où les quantités de nutriments dans les feuilles, et tout particulierement pour l'azote, sont toujours plus importantes à ce niveau d'altitude.

Les courbes théoriques des figures 2- 6 montrent des différences significatives dans les réponses des éléments minéraux aux deux niveaux altitudinaux. Ces différences ne sont pas seulement quantitatives mais concernent aussi la silhouette particulière de chacune de ces courbes. Ainsi, l'azote montre une absence de décroissance des teneurs en fin d'année dans les stations alticoles. L'évolution du potassium offre un profil original dans les stations thermophiles.

Au plan édaphique, le tableau II et les données recueillies ne permettent pas de justifier ces différences. Le gradient climatique important influence certainement la diversité phénologique. Cependant cela n'explique pas la variation tardive de la teneur en azote dans les stations thermophiles, plus précoces. En revanche, cela pourrait justifier le profil de la courbe du potassium dans ces mêmes stations, rappelant alors celui des feuillages d'arbres à feuilles persistantes.

Ceci pourrait constituer un exemple de l'adaptabilité du chêne zeen aux conditions d'un bioclimat plus propice au chêne-liège. La plus grande diversité des réponses dans la zone alticole peut être expliquée par l'impact de la saison sèche estivale sur le bilan de certains nutriments mais aussi par l'effet de l'anthropisation dans le couvert forestier.

Au plan écophysiologique, $Q$ canariensis est plus sensible aux perturbations dans sa zone de développement naturel que dans la zone de développement de son concurrent direct $Q$ suber. Dans cette dernière situation, il s'implante toujours dans des secteurs privilégiés (comme les fonds de vallon) et semble alors moins vulnérable aux perturbations liées à l'ouverture du milieu forestier.

Le diagnostic foliaire effectué sur $Q$ canariensis permet d'élargir le champ d'investigation écologique de manière significative. La concordance entre les analyses multivariées sur les données écologiques et sur les données relatives au bilan des nutriments dans les feuilles au cours d'un cycle annuel en est un exemple significatif.

Ce profil type annuel des nutriments considérés dans les feuilles de $Q$ canariensis ne pourra être établi que par comparaison avec celui d'autres essences forestières actuellement en cours d'étude.

Si l'on compare les taux de nutriments de $Q$ canariensis avec ceux du chêne-liège en Tunisie (dans la même région et à la même période) ou ceux de $Q$ rubra en France, on peut noter chez le chêne zeen des valeurs plus importantes en magnésium $(2,5 \mathrm{mg} / \mathrm{g}$ contre 2,1 chez $Q$ suber et 0,6 chez $Q$ rubra), et en potassium $(8,5 \mathrm{~m} / \mathrm{g}$ contre 7,0 chez $Q$ suberet 4,7 chez $Q$ rubra), mais les deux espèces de chênes tunisiens présentent des taux d'azote nettement plus faibles que ceux de $Q$ rubra (11 $\mathrm{mg} / \mathrm{g}$ contre 36 ) en Europe.

Enfin, il convient de souligner aussi la difficulté d'interprétation des résultats sur le territoire tunisien, compte tenu de l'impact très important de l'anthropisation sur le milieu forestier. Or, comme l'a montré Livrelli (1993), les bilans nutritionnels effectués par le diagnostic foliaire sont très influencés par l'état de perturbation des arbres. 


\section{RÉFÉRENCES}

Aime S, Bonin G, Chaabanne A, Loisel R, Saoudi H (1986) Notes phytosociologiques nord-africaines. Contribution à l'étude phytosociologique des zeenaies du littoral algéro-tunisien. Ecol Mediterranea 12, 113-131

Boxman AW, Cobben PLW, Roelofs JGM (1994) Does $(\mathrm{K}+\mathrm{Mg}+\mathrm{Ca}+\mathrm{P})$ fertilisation lead to recovery of tree health in a nitrogen stressed Quercus rubra L stand? Environ poll $85,297-303$

Charlot G (1978) Dosages absorptiométriques des éléments minéraux. Masson, Paris

Ed Derfoufi F (1986) Gestion et dynamique des nutriments dans des taillis de chênes verts âgés et très jeunes. Thèse de $3^{\mathrm{e}}$ cycle, Montpellier

Garbaye J (1972) Influence de la date et de la hauteur du prélèvement sur les résultats de l'analyse foliaire chez deux clones de peuplier. Ann Sci For 29, 451463

Gautier $P$ (1968) Vingt ans de diagnostic foliaire dans un verger de pommiers en Sologne. C R Acad Agric Fr 493-503

Janin G, Clément A (1972) Mise en évidence de cristaux de carbonate de calcium dans le bois des peupliers. Conséquences sur la répartition des ions minéraux liés à la duraminisation. Ann Sci For 29, 67-105

Lagatu $H$, Maume $L$ (1929) Le diagnostic foliaire et son degrè de sécurité. C R Acad Sci 188, 1062

Leroy $P$ (1968) Variations saisonnières des teneurs en eau et éléments minéraux des feuilles de chêne (Quercus pedunculata). Ann Sci For 25, 83-117

Le Tacon $F$, Toutain $F(1973)$ Variations saisonnières et stationnelles de la teneur en éléments minéraux des feuilles de hêtre (Fagus sylvatica) dans l'est de la France. Ann Sci For 30, 1-29

Levy JF (1968) L'application du diagnostic foliaire à la détermination des besoins alimentaires des vignes. $2^{*}$ Coll Europ et Médit Contr Fertil PI Cult 295-305

Livrelli JN (1993) Utilisation de la méthode du diagnostic foliaire chez des végétaux ligneux sclérophylles méditerranéens pour l'étude comparée de groupements préforestiers et forestiers. Thèse, université de Provence

Lowry GL, Avad PM (1969) Nutrient content of black spruce needles. I. Variation due to crown position and needle age. Pulp Paper Res Inst Can (1965). II. Variations with crown class and relationship to growth. Paper Res Inst Can (1968). III. Seasonal variations and recommanded sampling procedure. Paper Res Inst Can (1969)

Prevot P, Ollagnier M (1956) Méthodes d'utilisation du diagnostic foliaire. Anal Plant Prob Fum Mineral 2, 177-192

Quezel P, Bonin G (1980) Les forêts feuillues du pourtour méditerranéen : constitution, écologie, situations actuelles, perspectives. Rev For Fr 32, 253260

Smith PF (1962) Mineral analysis of plant tissues. Ann Rev Plant Physiol, 13, 81-108

Touzet G (1973) Analyse foliaire de l'épicéa et diagnostic des carences, IUFRO, Symp Internat sur la fertilisation forestière. Paris

Touzet G (1984) Essences forestières tempérées. In : L'analyse végétale dans le contrôle de l'alimentation des plantes tempérées et tropicales (P Martin Prével, J Gagnard, P Gauthier, P Lavoisier, eds), Paris, 525-537 